\title{
The analogy between heat and mass transfer in low temperature crossflow evaporation
}

\author{
Reza Enayatollahi*, Roy Jonathan Nates, Timothy Anderson \\ Department of Mechanical Engineering, Auckland University of Technology, \\ Auckland, New Zealand. \\ Corresponding Author: Reza Enayatollahi \\ Email Address: renayato@aut.ac.nz \\ Postal Address: WD308, 19 St Paul Street, Auckland CBD, Auckland, New Zealand \\ Phone Number: +6499219999 x8109
}

\begin{abstract}
This study experimentally determines the relationship between the heat and mass transfer, in a crossflow configuration in which a ducted airflow passes through a planar water jet. An initial exploration using the Chilton-Colburn analogy resulted in a coefficient of determination of 0.72 . On this basis, a re-examination of the heat and mass transfer processes by Buckingham's- $\pi$ theorem and a least square analysis led to the proposal of a new dimensionless number referred to as the Lewis Number of Evaporation. A modified version of the Chilton-Colburn analogy incorporating the Lewis Number of Evaporation was developed leading to a coefficient of determination of 0.96 .
\end{abstract}

\section{Introduction}

Heat and mass transfer devices involving a liquid interacting with a gas flow have a wide range of applications including distillation plants, cooling towers and aeration processes and desiccant drying [1-5]. Many studies have gone through characterising the heat and mass transfer in such configurations [6-9]. The mechanisms of heat and mass transfer are similar and analogical. Therefore, in some special cases where, either the heat or mass transfer data are not reliable or may not be available, the heat and mass transfer analogy can be used to determine the missing or unreliable set of data. In this regards, the Reynolds analogy is the simplest correlation and is applicable only for the special case where the Prandtl and Schmidt numbers are both equal to unity. Chilton and Colburn in 1934 [10] introduced a correlation to predict the coefficient of mass transfer from the experimental data of heat transfer and fluid friction, which is applicable for fully developed flow inside the tubes or between parallel plates with; $0.6<$ Prandtl $<60$ and $0.6<$ Schmidt $<3000$.

However, both of these analogies characterise the "convectional" transport phenomena and may not be applicable for some special cases and geometries. Therefore a number of studies have examined the applicability of these analogies to other configurations [11-13]. Steeman et al. [12] employed CFD to investigate the validity of the heat and mass transfer analogy for a particular case of indoor airflows and when the analogy conditions are not met. Similarly, Tsilingiris [14] experimentally developed a heat and mass transfer analogy model in solar distillation systems based on the Chilton-Colburn analogy.

This study investigates the analogy between the intensities of heat and mass transfer in low temperature evaporation processes with crossflow configuration, in which a ducted stream of air passes through a falling sheet of water. The interaction in such a configuration has the potential to significantly improve the transfer phenomenon. 


\section{Experimental Setup}

In this experiment, a planar jet of water was directed perpendicular to a ducted air crossflow, as shown in Figure 1. A water tank with adjustable height was used to provide a constant pressure head to drive the water flow at different flow rates and a variable speed axial flow fan with a maximum capacity of $280 \mathrm{~m}^{3} / \mathrm{hr}$ was employed to drive airflow at various steady flow rates. The flow rates of water were determined by measuring the time taken for a known volume of water to pass through the nozzle, and the exact airflow rate was determined from measurements made using a pitot static probe traversed across the duct and differential manometer.

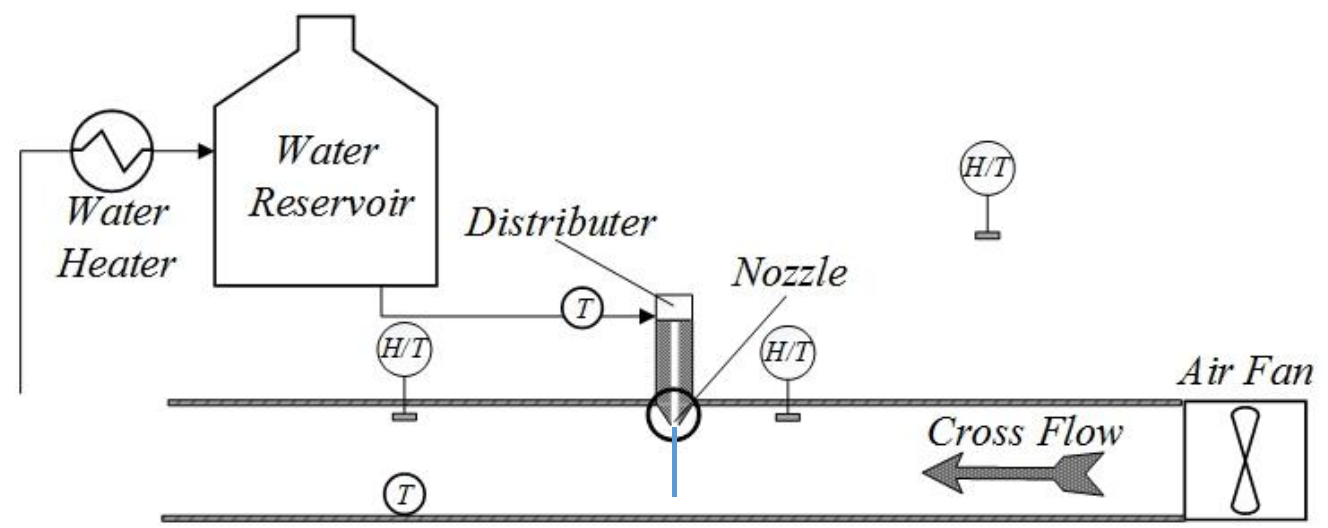

Figure 1. Experimental apparatus

In order to measure the humidity and temperature, a set of three humidity/temperature sensors, (Sensirion SHT71) with an accuracy of $\pm 3 \%$ for humidity and $\pm 0.3 \mathrm{~K}$ temperature at standard room condition were used. Sensors were placed on either side of the side the sheet to measure the change in humidity and temperature of the air stream as it crossed the water sheet, as seen in Figure 1. A third sensor was placed outside the experiment to monitor the room conditions. A set of two thermocouples (type T) with an accuracy of $\pm 0.3 \mathrm{~K}$ were used to record the water temperature before and after contact with the air stream. An auxiliary water heater was used to maintain the inlet water temperature at a constant temperature and thereby reduce the relative error of measurements.

\section{Testing the Chilton-Colburn Analogy}

In considering the heat transfer, the total rate of heat transfer $\left(\dot{Q}_{t}\right)$ is the sum of convective, evaporative and radiative rates of heat transfer. Assuming that the radiation heat transfer is negligible this can be determined from Equation 1.

$$
\dot{Q}_{t}=\dot{Q}_{c v}+\dot{Q}_{e v}=\dot{m}_{a}\left(\hbar_{a, o}-\hbar_{a, i}\right)
$$

Where $\dot{Q}_{c v}$ is the convective rate of heat transfer and $\dot{Q}_{e v}$ is the rate of heat transfer through evaporation. $\dot{m}_{a}$ is the mass flow rate of air and $\hbar_{a, i}$ and $\hbar_{a, o}$ are the enthalpies of the air at the inlet and outlet conditions, respectively. The rate of evaporation can be determined from Equation 2.

$$
\dot{Q}_{e v}=\dot{m}_{e v} \hbar_{f g}
$$


Where $\hbar_{f g}$ is the enthalpy of vaporization and $\dot{m}_{e v}$ is the rate of evaporation, which can be calculated by measuring the specific humidity $(\omega)$ of air at inlet and outlet conditions and the mass flow rate of the air stream as given in Equation 3.

$$
\dot{m}_{e v}=\dot{m}_{a}\left(\omega_{a, o u t}-\omega_{a, \text { in }}\right)
$$

On the mass transfer side the experimental value of the coefficient of mass transfer can be determined from Equation 4.

$$
j=\frac{\dot{m}_{e v}}{A_{c, a}\left(\rho_{v, f}-\rho_{v, b}\right)}
$$

Where, $\rho_{v, \infty}$ is the density of vapour at the free stream conditions and $\rho_{v, f}$ is the vapour density at film condition, which is considered to be saturated air at the average temperature of the two phases.

The experimental value of the coefficient of convective heat transfer can be calculated from Equation 5.

$$
h=\frac{\dot{Q}_{c v}}{A_{c, a}\left(T_{f}-T_{\infty}\right)}
$$

Where $A_{c, a}$ is the cross sectional area of air stream, $T_{\infty}$ is the bulk stream temperature and $T_{f}$ is the film temperature. The convective heat transfer rate can be determined from Equation 1 .

The existence of an analogy was first assessed by examining the relationship between the heat transfer coefficient determined from Equation 5 and the mass transfer coefficient calculated by Equation 4, as shown in Figure 2. 


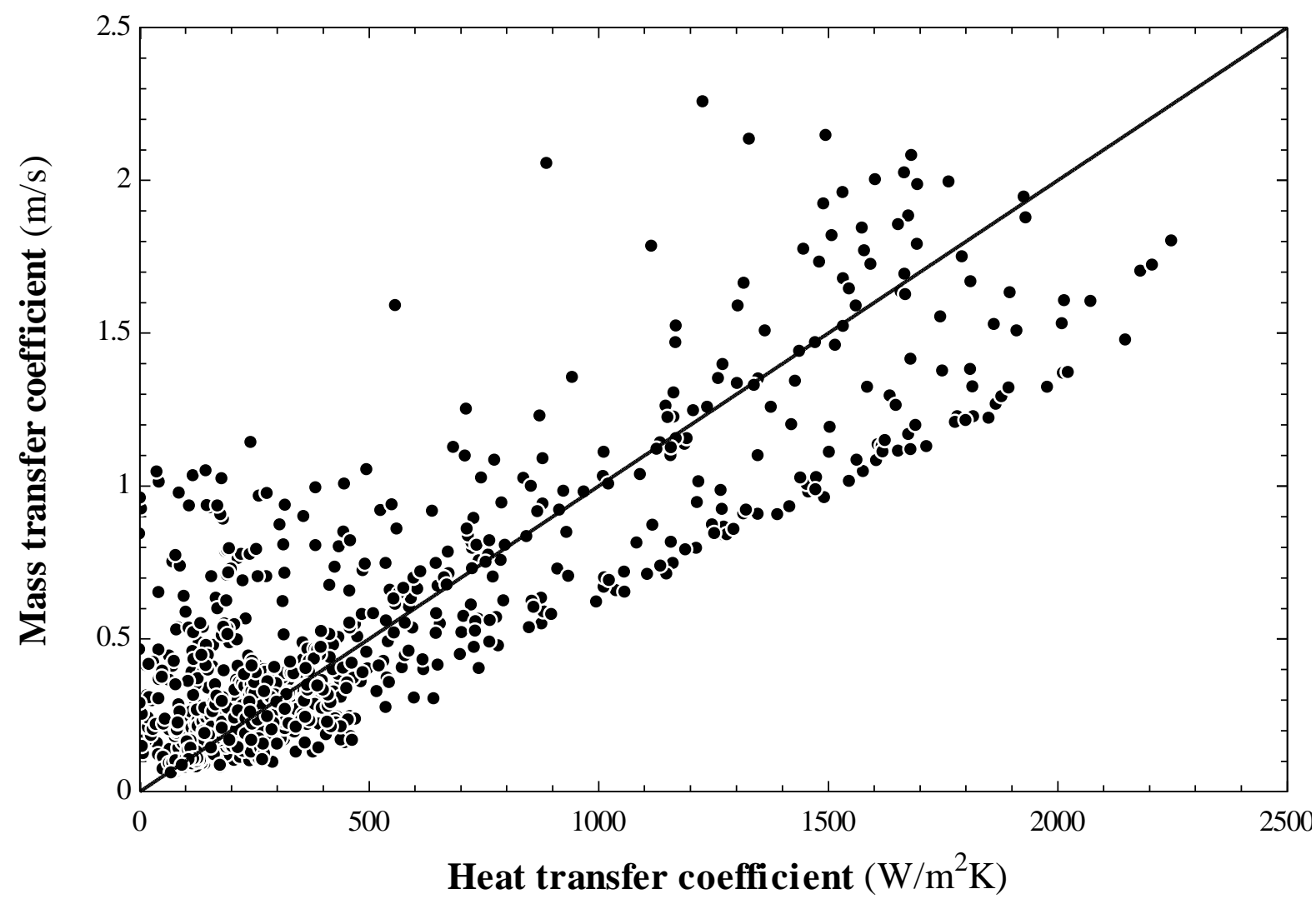

Figure 2. The experimental values of convective heat transfer coefficient versus experimental values of mass transfer coefficient

As seen in Figure 2, the experimental values of the heat and mass transfer coefficients are correlated with a reasonable accuracy, with a coefficient of determination $\left(\mathrm{R}^{2}\right)$ of 0.72 . The heat and mass transfer are analogues, in circumstances where the thermal and concentration boundary layers are of the same type [15]. For the conditions tested by Chilton and Colburn, the empirical correlations of Nusselt and Sherwood numbers were determined as given in Equations 6 and 7 [16].

$$
\begin{aligned}
& N u=a \operatorname{Re}^{m} \operatorname{Pr}^{1 / 3} \\
& S h=a R e^{m} S c^{1 / 3}
\end{aligned}
$$

Based on the Reynolds analogy the heat transfer Stanton number is equivalent to the mass transfer Stanton number. Where the heat transfer Stanton number is the ratio of the Nusselt number to the product of the Reynolds and Prandtl numbers, and the mass transfer Stanton number is the ratio of the Sherwood number to the product of the Reynolds and Schmidt numbers, as given in Equations 8 and 9 [15].

$$
\begin{gathered}
S t_{\text {heat }}=\frac{h}{\rho V c_{p}}=\frac{N u}{R e P r} \\
S t_{\text {mass }}=\frac{j}{V}=\frac{N u}{R e P r}
\end{gathered}
$$

Now, substituting the empirical correlation for the Nusselt and Sherwood numbers, results in Equations 10 and 11. 


$$
\begin{gathered}
S t_{\text {heat }}=\frac{h}{\rho V c_{p}}=\frac{a R e^{m} P r^{1 / 3}}{\operatorname{Re} P r} \\
S t_{\text {mass }}=\frac{j}{V}=\frac{a R e^{m} S c^{1 / 3}}{\operatorname{Re} S c}
\end{gathered}
$$

From these, Chilton and Colburn had derived a " $J$ " factor for heat and mass transfer as given in Equations 12 and 13 [10].

$$
\begin{gathered}
J_{\text {heat }}=a R e^{m-1}=\frac{h}{\rho V c_{p}} \operatorname{Pr}^{2 / 3} \\
J_{\text {mass }}=a R e^{m-1}=\frac{j}{V} S c^{2 / 3}
\end{gathered}
$$

Since the " $J$ " factor is equal for both heat and mass transfer, the Chilton-Colburn analogy was determined as given in Equation 14 [10].

$$
\begin{gathered}
\frac{h}{\rho V c_{p}} \operatorname{Pr}^{\frac{2}{3}}=\frac{j}{V} S c^{\frac{2}{3}} \\
\text { or } \quad \frac{h}{j}=\rho c_{p}\left(\frac{S c}{\operatorname{Pr}}\right)^{2 / 3}=\rho c_{p} L e^{2 / 3}
\end{gathered}
$$

As mentioned earlier the Chilton-Colburn analogy, seen in Equation 14, is valid for a fully developed flow inside a pipe, and for flow parallel to plane surfaces, when $0.6<$ Prandtl $<60$ and $0.6<$ Schmidt $<3000$.

The applicability of the Chilton-Colburn analogy to other configurations and conditions may be validated for the particular geometry and conditions of the experiment.

Figure 3 shows the experimental values of the convection heat transfer coefficient from Equation 5 compared to the calculated value from the Chilton-Colburn analogy, given in Equation 14 using the experimental mass transfer data. This figure shows some correlation for predicting the heat transfer coefficient from the mass transfer data, but with quite a large scatter. 


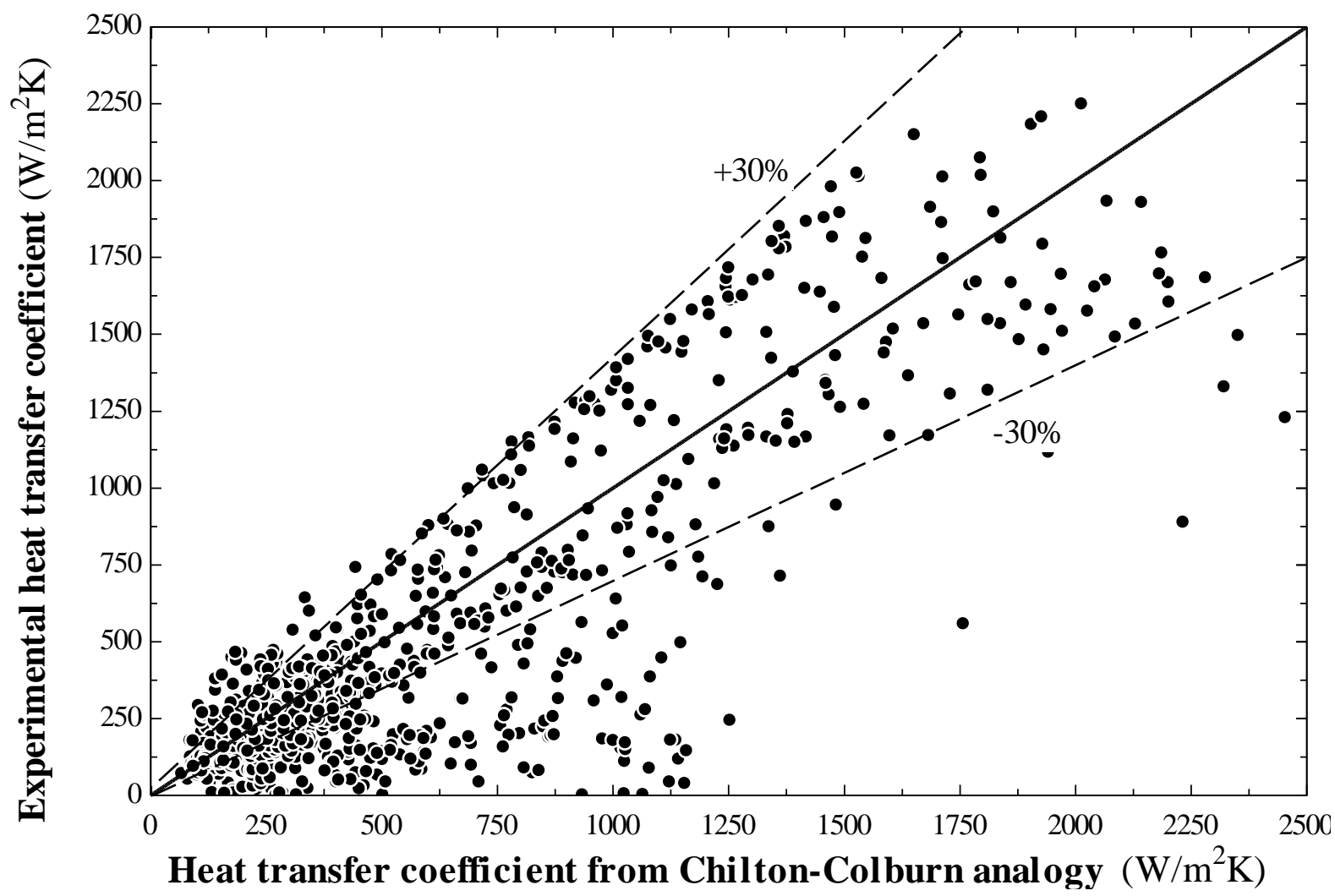

Figure 3. Comparison of experimental heat transfer coefficient with the calculated values from the Chilton-Colburn analogy

From this, it could be considered that, the Chilton-Colburn analogy is reasonably valid for these geometries and conditions. However, considering that in low temperature evaporation processes a considerable fraction of the supplied energy will be consumed to overcome the latent heat of vaporization, it is reasonable to expect that the relationship between heat and mass transfer should account for this. Therefore, this work aims to find an analogy between the overall heat transfer coefficient and mass transfer coefficient, in that it accounts for both convection and evaporation.

\section{Dimensional analysis}

In an attempt to describe the analogy between heat and mass transfer, for this complex flow interaction, Buckingham's- $\pi$ theorem was employed to define the dimensionless parameters. In doing so it was considered that the variables describing the heat and mass transfer were: air velocity, characteristic length, enthalpy of evaporation, thermal conductivity of air, density of air, viscosity of air, specific heat of air, rate of diffusion, the enthalpy content of the air stream at film conditions and the temperature difference between two phases. As such a general relation as shown in Equation 15 can be formed.

$$
f\left(\rho_{a}, k_{a}, V_{a}, L_{a}, h_{h}, \mu_{a}, c p_{a}, \Delta T, D_{A B}, h_{m}, h_{f g}, T_{f}\right)=0
$$

where $f$ is an unknown function. The dimensions of these variable are based on four basic physical units of mass (M), temperature $(\mathrm{T})$, time $(\mathrm{t})$ and length $(\mathrm{L})$.

As there are twelve quantities and four basic units, according to Buckingham's- $\pi$ theorem [17], eight dimensionless groups can be developed, as shown in Equation 16. 


$$
f^{\prime}\left(\pi_{1}, \pi_{2}, \pi_{3}, \pi_{4}, \pi_{5}, \pi_{6}, \pi_{7}, \pi_{8}\right)=0
$$

Where $f^{\prime}$ is also an unknown function. Choosing $\rho_{a}, k_{a}, V_{a}$ and $L_{a}$ as the repeating parameters, the seven independent dimensionless group can be determined as given in Table 1.

Table 1. Independent dimensionless groups

\begin{tabular}{|c|c|c|c|}
\hline$\pi_{1}=\frac{L_{c h} h}{k_{a}}$ & $\pi_{3}=\frac{\rho_{a} V_{c h} L_{c h} c p_{a}}{k_{a}}$ & $\pi_{5}=\frac{\lambda_{a-w}}{V_{c h} L_{c h}}$ & $\pi_{7}=\frac{\hbar_{f g}}{V_{c h}{ }^{2}}$ \\
\hline$\pi_{2}=\frac{\mu_{a}}{\rho_{a} V_{c h} L_{c h}}$ & $\pi_{4}=\frac{k_{a} \Delta T}{\rho_{a} V_{c h}{ }^{3} L_{c h}}$ & $\pi_{6}=\frac{j}{V_{c h}}$ & $\pi_{8}=\frac{\hbar_{f}}{V_{c h}{ }^{2}}$ \\
\hline
\end{tabular}

Where, $\pi_{1}$ is the Nusselt number $(N u)$ and product of $\pi_{6}$ and $\pi_{5}^{-1}$ forms the Sherwood number $(S h), \pi_{2}^{-1}$ is the Reynolds number $(\mathrm{Re}), \pi_{3}$ is the Peclet number $(P e)$ and $\pi 7^{-1}$ is the Evaporation number $\left(N_{e v}\right)$. Grouping $\pi_{2}$ and $\pi_{3}$ delivers the Prandtl number $(P r)$ and combination of $\pi_{2}$ and $\pi_{5}$ gives the Schmidt number $(S c)$. The Lewis number $(L e)$ can also be determined as the ratio of Prandtl to Schmidt number, which is the ratio of thermal to mass diffusivity.

\section{Analogy between the Coefficient of Total Heat Transfer and Mass Transfer Coefficient}

As mentioned earlier, the Chilton-Colburn analogy characterises only the convectional transfer phenomenon and since in low temperature evaporation processes a significant fraction of the entire heat transfer is through evaporation, it is logical to present a correlation to predict the overall heat transfer coefficient from the mass transfer data. In this respect, the experimental value of the overall heat transfer coefficient can be calculated by substituting $\dot{Q}_{c v}$ in Equation 5 with $\dot{Q}_{t}$ as shown in Equation 17.

$$
h_{t}=\frac{\dot{Q}_{t}}{A_{c, a}\left(T_{f}-T_{\infty}\right)}
$$

In order to assess the existence of any similarity between the coefficient of total heat transfer and the mass transfer coefficient, the calculated values from Equation 17 were plotted against the experimental values of mass transfer coefficient from Equation 4, as shown in Figure 4. 


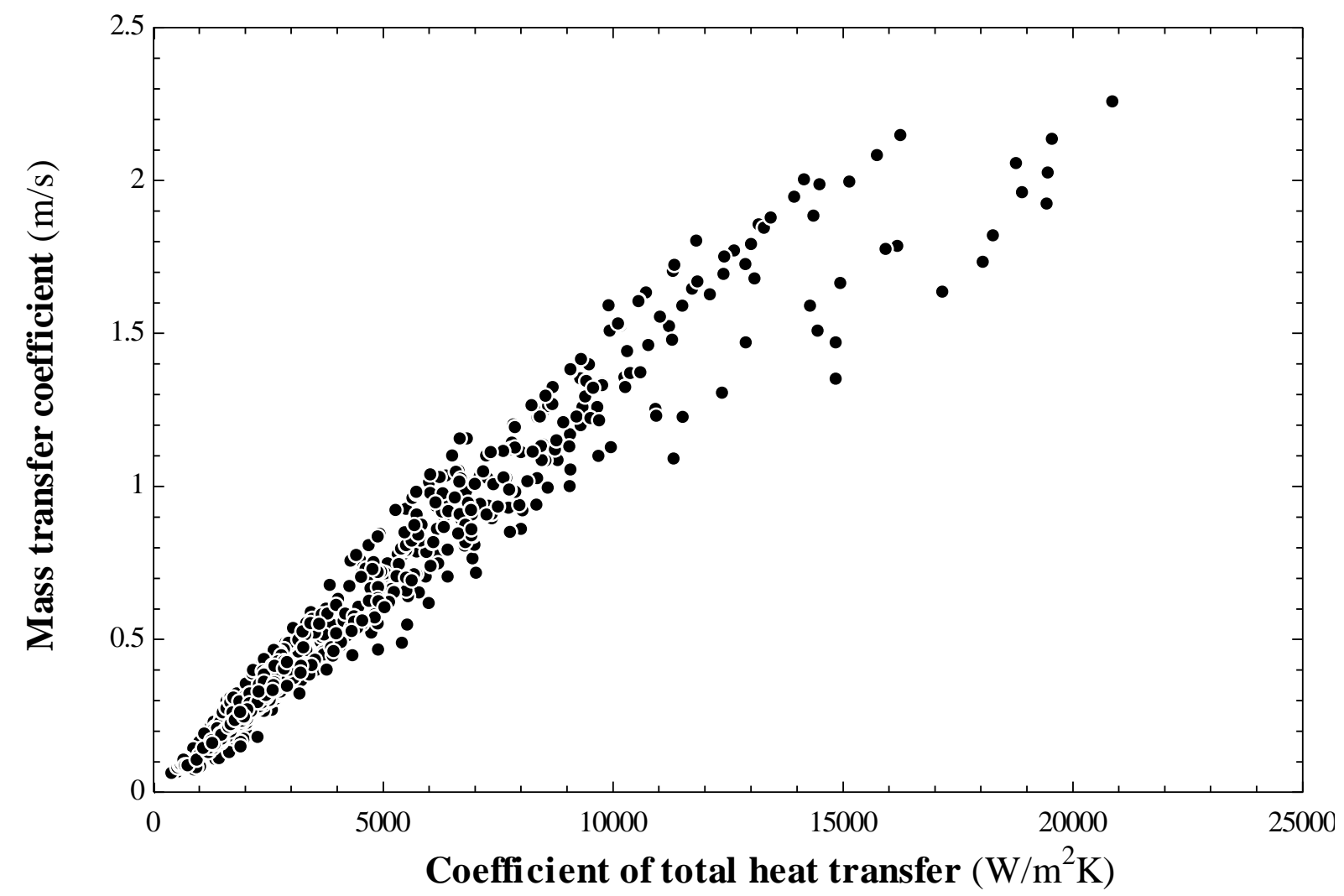

Figure 4. the coefficent total heat transfer versus the mass transfer coefficient

In Figure 4, it can be seen that the mass transfer coefficient is analogous with the coefficient of total heat transfer with a coefficient of determination $\left(\mathrm{R}^{2}\right)$ of 0.95 .

On this basis, the overall heat transfer coefficient can be expressed as a function of the density and the specific heat of the air stream as well as the dimensionless groups derived from Buckingham's $\pi$ theorem, as given in Equation 18.

$$
h_{t}=j \rho c_{p} \times P e^{b} B d^{c} \pi_{4}{ }^{d} \pi_{7}^{e} \pi_{8}^{f}
$$

In order to define the exponent of the dimensionless groups in Equation 18, a least squares analysis results in Equation 19.

$$
h_{t}=j \rho c_{p} \times P e^{-0.575} B d^{0.575} \pi_{4}^{0.00012} \pi_{7}^{0.575} \pi_{8}{ }^{-0.575}
$$

The exponents of the Peclet and Bodenstein numbers are identical but with different signs and therefore, can be presented in a fractional form. As mentioned earlier the Bodenstein is the product of the Reynolds and Schmidt numbers and similarly, the Peclet number is the product of the Reynolds and Prandtl numbers. Therefore, the ratio of Bodenstein to Peclet numbers is in fact the ratio of Schmidt to Prandtl number, or the Lewis number (Le), as given in Equation 20.

$$
\frac{B d^{0.575}}{P e^{0.575}}=\left(\frac{B d}{P e}\right)^{0.575}=\left(\frac{S c}{P r}\right)^{0.575}=L e^{0.575}
$$


Similarly, the exponents of $\pi 7$ and $\pi 8$ are identical but with opposite signs and can be presented in a fractional form, as given in Equation 21.

$$
\frac{\pi_{7}}{\pi_{8}}=\frac{\hbar_{f g} / V_{c h}^{2}}{\hbar_{f} / V_{c h}{ }^{2}}=\frac{\hbar_{f g}}{\hbar_{f}}
$$

The ratio of $\pi 7$ to $\pi 8$ is in fact the ratio of enthalpy of vaporization to the enthalpy content of the air stream at the film conditions, which in this context referred to as the enthalpy ratio. The enthalpy ratio characterises the low temperature evaporation processes with respect to required heat of evaporation and the supplied energy by the bulk stream.

It can be seen that the exponents of the Lewis number and the enthalpy ratio are equal and hence, Equation 19 can now be rewritten as Equation 22.

$$
h_{t}=j \rho_{a} c p_{a}\left(L e \frac{\hbar_{f g}}{\hbar_{f}}\right)^{0.575}
$$

The product of Lewis number and the enthalpy ratio is therefore referred to as the Lewis Number of Evaporation $\left(L e_{e v}\right)$ and Equation 22 can be rewritten as Equation 23.

$$
h_{t}=j \rho_{a} c p_{a} L e_{e v}^{0.575}
$$

Shown in Figure 5 is the coefficient of total heat transfer calculated by Equation 23 versus the experimental values calculated from Equation 17.

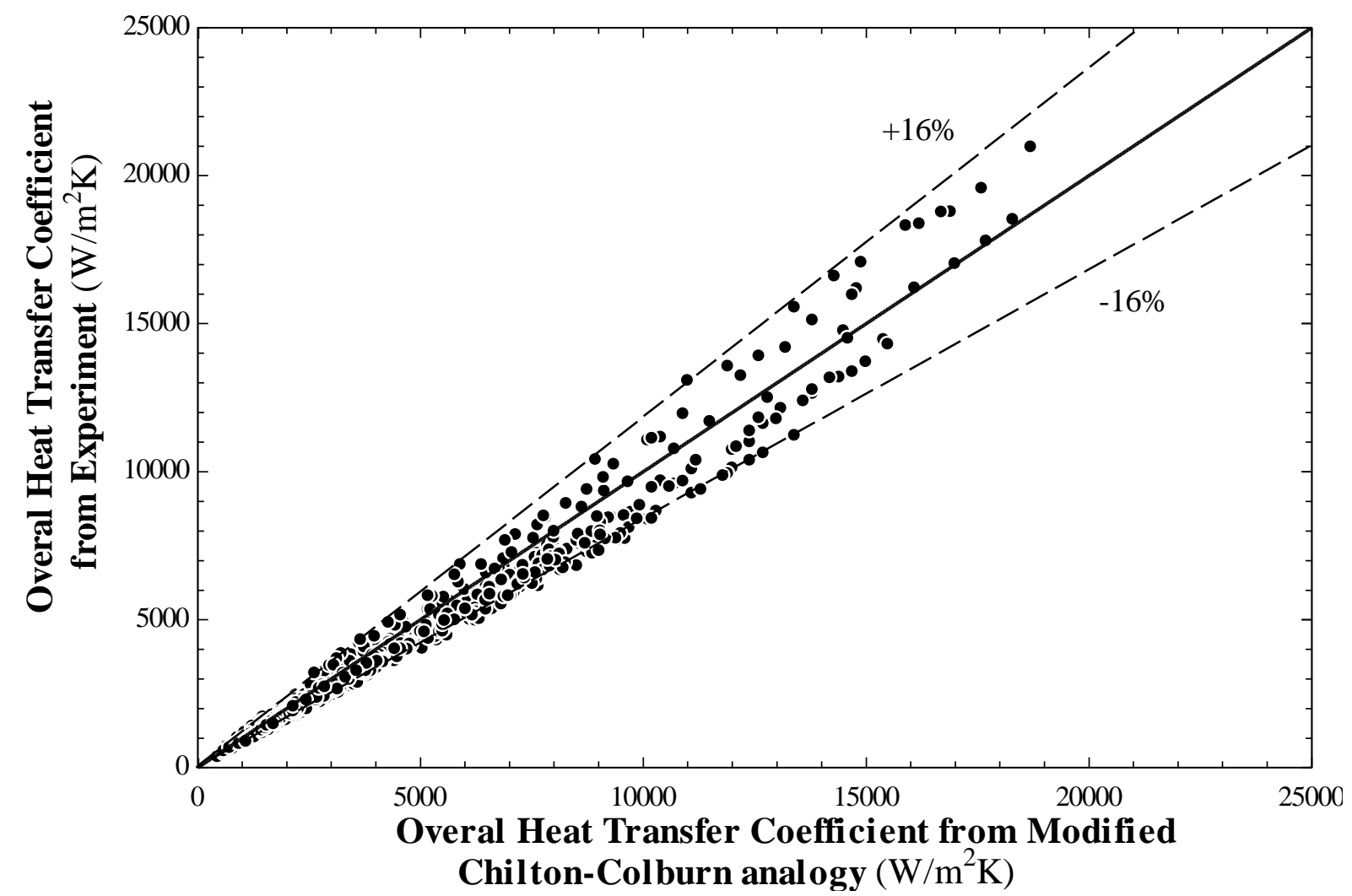

Figure 5. Corrected Chilton-Colburn Analogy for heat and mass transfer with phase change 
This figure clearly shows a much stronger correlation, with a coefficient of determination $\left(\mathrm{R}^{2}\right)$ of 0.98 , when accounting for the phase change process and incorporating the Lewis Number of Evaporation.

\section{Conclusion}

An experimental study was performed in order to examine the relationship between heat and mass transfer coefficients in a low temperature crossflow evaporation process. In this regard, the Buckingham- $\pi$ theorem as well as a least squares analysis were employed. Eight dimensionless group were determined from the Buckingham- $\pi$ analysis. Performing the least squares analysis on these dimensionless parameters showed a strong correlation between the overall heat transfer coefficient and the enthalpy ratio. This led to the correlation of a modified Chilton-Colburn analogy that includes the enthalpy ratio to account for the low temperature evaporation processes (referred to as the Lewis Number of Evaporation). As a result of this work, the heat and mass transfer can now be quantified by the measurement and determination of only one of these parameters.

\section{References}

[1] R. Enayatollahi, T. Anderson, R. Nates, Mathematical modeling of a solar powered humidification dehumidification desalination prototype, in: Solar 2014: The 52nd Annual Conference of the Australian Solar Council, Solar 2014 Conference \& Expo, Melbourne, 2014.

[2] V.D. Stevanovic, An analytical model for gas absorption in open-channel flow, International Communications in Heat and Mass Transfer, 24 (1997) 1187-1194.

[3] P. Naphon, Study on the heat transfer characteristics of an evaporative cooling tower, International Communications in Heat and Mass Transfer, 32 (2005) 1066-1074.

[4] A. Klimanek, R.A. Biatecki, , Solution of heat and mass transfer in counterflow wet-cooling tower fills, International Communications in Heat and Mass Transfer, 36 (2009) 547-553.

[5] Z. Huang, P. Jiang, Theoretical Analysis and Numerical Simulation of Coupled Relationship of Heat and Mass Transfer Between Air and Desiccant in Liquid Desiccant Dehumidification, in: Proceedings of the 8th International Symposium on Heating, Ventilation and Air Conditioning, Springer, 2014, pp. 829-839.

[6] A. Ali, K. Vafai, A.R.A. Khaled, Analysis of heat and mass transfer between air and falling film in a cross flow configuration, International Journal of Heat and Mass Transfer, 47(4) (2004) 743-755.

[7] D. Metzger, L. Florschuetz, D. Takeuchi, R. Behee, R. Berry, Heat transfer characteristics for inline and staggered arrays of circular jets with crossflow of spent air, Journal of Heat Transfer, 101(3) (1979) 526-531.

[8] Y. Yin, B. Zheng, T. Chen, B. Shao, X. Zhang, Investigation on coupled heat and mass transfer coefficients between compressed air and liquid desiccant in a packed dryer, International Journal of Heat and Mass Transfer, 93 (2016) 1218-1226.

[9] Y. Wu, Simultaneous heat and mass transfer in laminar falling film on the outside of a circular tube, International Journal of Heat and Mass Transfer, 93 (2016) 1089-1099. 
[10] T.H. Chilton, A.P. Colburn, Mass Transfer (Absorption) Coefficients Prediction from Data on Heat Transfer and Fluid Friction, Industrial \& Engineering Chemistry, 26(11) (1934) 1183-1187.

[11] D. Bond, M.J. Goldsworthy, V. Wheatley, Numerical investigation of the heat and mass transfer analogy in rarefied gas flows, International Journal of Heat and Mass Transfer, 85 (2015) 971-986.

[12] H.J. Steeman, A. Janssens, M. De Paepe, On the applicability of the heat and mass transfer analogy in indoor air flows, International Journal of Heat and Mass Transfer, 52(5-6) (2009) $1431-1442$.

[13] G. Caruso, D. Vitale Di Maio, Heat and mass transfer analogy applied to condensation in the presence of noncondensable gases inside inclined tubes, International Journal of Heat and Mass Transfer, 68 (2014) 401-414.

[14] P.T. Tsilingiris, The application and experimental validation of a heat and mass transfer analogy model for the prediction of mass transfer in solar distillation systems, Applied Thermal Engineering, 50(1) (2013) 422-428.

[15] Y.A. Cengel, Heat and Mass Transfer. 3rd, in, New York: McGraw-Hill, 2007.

[16] A.P. Colburn, A method of correlating forced convection heat-transfer data and a comparison with fluid friction, International Journal of Heat and Mass Transfer, 7(12) (1964) $1359-1384$.

[17] E. Buckingham, Model experiments and the forms of empirical equations, 1915. 